\title{
Learning pixel-wise signal energy for understanding semantics
}

\author{
Jeffrey $\mathrm{Ng}^{*}$, Shaogang Gong \\ Department of Computer Science, Queen Mary, University of London, London E1 4NS, UK
}

\begin{abstract}
Visual interpretation of events requires both an appropriate representation of change occurring in the scene and the application of semantics for differentiating between different types of change. Conventional approaches for tracking objects and modelling object dynamics make use of either temporal region-correlation or pre-learnt shape or appearance models. We propose a new pixel-level approach for learning the temporal characteristics of change at individual pixels. Gaussian mixture models are used to model slow long-term changes in pixel distributions while pixel energy histories are used to extract fast-change signatures from short-term events and modelled by CONDENSATION matching.

(C) 2003 Elsevier B.V. All rights reserved.
\end{abstract}

Keywords: Gaussian mixture models; Deviant-event; Quadrature filter pair; Phase information; CONDENSATION-based trajectory matching; Abnormal event detection

\section{Introduction}

In visual surveillance, automated systems are confronted with environments under constant change. For such dynamic scenes, visual change is not necessarily an indication of the occurrence of problematic events but rather, is a function of the context (semantics) of the scene. Deviations from established patterns of change in the image may signal an abnormal event under way. For example, constant rapid motion can be observed on a busy road and a sudden absence of motion may reveal an accident, while rapid motion on the sidewalk areas which have previously only been used by slower moving pedestrians is likely to imply abnormal behaviour.

Previous works addressed the problem of sceneinterpretation by explicitly modelling change in terms of the dynamics of moving objects. Object detection and tracking have been performed by numerous methods such as colour object models [11] and background subtraction $[9,12]$, while the trajectories of moving objects have been modelled using Kalman filters [12] and augmented Hidden Markov densities [8]. However, appearance models are difficult to obtain in unconstrained environments such as shopping malls. And the application of region-growing techniques on collections

\footnotetext{
* Corresponding author. Tel.: +44-207-882-5200; fax: +44-207-8826533.

E-mail address: jeffing@dcs.qmul.ac.uk (J. Ng).
}

of pixels obtained from background subtraction artificially induces spatial correlations which complicates the disambiguation process for object or group-based trajectory event recognition in free-flowing group-based behaviours.

It is argued here that temporal information contained in the colour signal of individual pixels constitutes a more attractive alternative for understanding events than spatial connectivity or proximity. Pixel signal energy, computed from the local colour history of the pixel, provides a condensed temporal measure of change. Although the latter is related to computing visual motion such as optical flow from motion-energy filters [6], we are not interested in establishing correspondence in local pixel neighbourhoods. Rather, we are only interested in extracting reliable temporal change at individual pixels. We then consider that meaningful events rather than simply motion in the image sequence should be modelled through understanding the energy-history of each individual pixel. This is to some extent reminiscent of the notion of 'topic spotting' in speech recognition, i.e. extracting meaning without explicitly modelling the details. Furthermore, pixel energy constitutes a good measure for exploiting synchrony in pixel-events, which have been extensively researched in the psychophysical literature [13]. Synchronous recognition of pixelevents addresses the limitation of the short-sighted view of single pixels in the scene and provides a more flexible framework for understanding global events as opposed to related spatio-temporal motion-energy measures [1]. 
To cope with different types of pixel-change, we propose a two-stage scheme. In Section 2, we make use of adaptive Gaussian Mixture Models (GMM) for modelling long-term colour distributions of pixels, especially slow change caused by lighting cycles. While GMMs provide the platform for long-duration scene analysis, they have also been probabilistically formulated for detecting faster short-term change to perform more computation intensive synchronous energy-history recognition. A novel approach is proposed in Section 3 involving energy histories of pixel-change for CONDENSATION-based recognition. Finally, experimental results are provided in Section 4 to investigate the relationship between low-level energy information and high-level semantics for understanding scene events. The technique is also compared to traditional GMM background modelling.

\section{Detecting change}

Dynamic scenes exhibit a wide spectrum of change both in terms of the speed and nature of the change occurring in individual pixels. Fast short-term change can be caused by a variety of objects of unknown appearance and is mostly characterised by the dynamics of the change and thus its temporal profile. On the other hand, long-term change causes slow shifts in the colour distribution of the scene background seen at the pixel. Whether these components are generated by scintillating static objects in the background or cyclically moving objects, they can be modelled by Gaussian mixture models. More specifically, given a stream of colour values for a given pixel, $\mathbf{x}_{t} \in\left\{\mathbf{x}_{0}, \mathbf{x}_{1}, \ldots, \mathbf{x}_{l}\right\}$, the variation in the $(r, g, b)$ components of $\mathbf{x}_{t}$ can be described in terms of Gaussian means $\mu$ and covariances $\Sigma$. Illumination specularities or swaying objects such as plants induce multiple modes into the colour distributions of pixels $[11,12]$, which require multiple Gaussian components. A Gaussian mixture $p(\mathbf{x})=\sum_{i=1}^{k} \omega_{i} \cdot \psi\left(\mathbf{x}, \mu_{i}, \Sigma_{i}\right)$ can be used, where $\omega_{i}$ represents the mixing parameter and $\psi(\cdot)$ the Gaussian kernel.

In unconstrained environments, the colour distributions of specific pixels rarely remain static. Changes in the lighting conditions or the patterns of sway of objects cause slow shifts in the parameters of the mixture models. First, we make these parameters adaptive in a similar fashion to the online approximation technique described in [12]. New visual evidence is approximated with uniform Gaussian clusters of pre-set variance according to the amount of noise present in the particular capturing setup. Offline methods such as $k$-means clustering or EM are not fast enough for computing thousands of mixtures for separate pixels. The clusters are then adapted to the particular distribution of the pixels.

For a new pixel $\mathbf{x}_{t}$, the closest Gaussian with Mahalanobis distance smaller than 2 s.d. ( $\sim 98 \%$ confidence) is selected as responsible. A learning rate $\alpha$ is used to constrain the pace of change of the means and covariance of the Gaussian as well as the mixture parameter $\omega$ of all the Gaussians to promote long-term changes of the distribution over short-term variation

$\mu_{t}=(1-\alpha) \mu_{t-1}+\alpha \mathbf{x}_{t}$

$\Sigma_{t}=(1-\alpha) \Sigma_{t-1}+\alpha\left(\mathbf{x}_{t} \cdot \mathbf{x}_{t}^{T}\right)$

$\omega_{u, t}=(1-\alpha) \omega_{u, t-1}+\alpha\left(M_{u, t}\right)$

$M_{u, t}= \begin{cases}1, & \text { if } u \text { is the responsible Gaussian } \\ 0, & \text { otherwise }\end{cases}$

A confidence factor $T$ is used to identify predominant components in the distribution and is expressed in terms of the overall ratio (in the range $[0,1]$ ) of predominant clusters. The Gaussian components in the mixture are ordered according to the product of (a) their weights, which reflect observation frequency and (b) the inverse of their variances to promote static objects with smaller variances. The first $b$ Gaussians which account for a proportion $T$ of observations, weighted by the inverse of their variance, are considered as predominant

$b=\underset{B=1}{k_{\max }}\left\{\sum_{i=1}^{B} \omega_{i}>T\right\}$

New clusters are generated for observations $\mathbf{x}_{t}$ which do not fit current clusters. Once a limit $k_{\max }$ is exceeded, the weakest, less important, cluster is replaced for computational reasons. In our case, we use $k_{\max }=6$ so that the mixture model mostly captures static components responsible for slow change.

We then formulate the probability of pixel values $\mathbf{x}_{t}$ belonging to pre-learnt set of long-term Gaussian clusters as opposed to recent foreground components by exploiting the total conditional probability stored in the Gaussian mixture model, instead of using only simple Mahalanobis distance to the closest Gaussian as in [12]

$P\left(\right.$ long-term $\left.\mid \mathbf{x}_{t}\right)=\frac{\sum_{i=1}^{b} p\left(\mathbf{x}_{t} \mid i, t\right) P(i, t)}{p\left(\mathbf{x}_{t}\right)}, \quad$ where

$p\left(\mathbf{x}_{t} \mid i, t\right)=\frac{1}{2 \pi^{3 / 2}\left|\mathbf{\Sigma}_{i, t}\right|^{1 / 2}}$

$$
\times \exp \left(-\frac{1}{2}\left(\mathbf{x}_{t}-\boldsymbol{\mu}_{i, t}\right)^{T}\left(\boldsymbol{\Sigma}_{i, t}\right)^{-1}\left(\mathbf{x}_{t}-\boldsymbol{\mu}_{i, t}\right)\right)
$$

$P(i, t)=\omega_{i, t}$

The configuration of the predominant set stores the accumulated history of the observation frequency of each component in the mixture over a long time scale. The state of the set can therefore capture slow changes in the colour distribution of pixels. Depending on the surveillance task, the predominant set can be locked so that new clusters are reported as abnormal, e.g. the introduction of a parcel in a busy scene. In the short term, the long-term models can detect and segment out interesting non-fitting fast-change 
which are subsequently modelled with energy histories in Section 3 .

\section{Recognising meaningful change}

Rapidly changing visual phenomena exhibited by the motion of animated objects typically involve both non-rigid deformations [9] and purposeful trajectories [5,8,12]. Illumination specularities further complicate the task of understanding scenes from purely visual data. Without higher-level knowledge provided in the form of pre-learnt object and trajectory models, it is very difficult to interpret frame-wise data. Indeed, semantics used for understanding scenes and classifying events operate on object-level information, which is not readily available in low-level pixel data. However, the temporal sequence of change in pixel data can provide a better cue as to the type of event occurring at the pixel's location. Pixel energy extracts the signature of change occurring at any time instant. Furthermore, we propose that histories, or temporal sequences, of pixel energy provide a generic means of extracting signatures from short-term visual change. Fig. 1 shows pixel energy collected from a sample sequence of a person moving from left to right and back.

Pixel energy Pe can be measured from the response of pairs of quadrature filters of temporal width $v$ [10] and filter cut-off $\mathrm{ct}=3.5$ where the filter coefficients become negligible

$\operatorname{Pe}\left(\mathbf{x}_{t}\right)=\left[\sum_{i=0}^{2 \nu} g\left(\frac{\operatorname{ct}(i-\nu)}{\nu}\right) \mathbf{x}_{t-i}\right]^{2}+\left[\sum_{i=0}^{2 \nu} h\left(\frac{\operatorname{ct}(i-\nu)}{\nu}\right) \mathbf{x}_{t-i}\right]^{2}$

The filter masks $g(y)$ and $h(y)$ are, respectively, defined as

$g(y)=\eta\left(2 y^{2}-1\right) \mathrm{e}^{-y^{2}}$

$h(y)=\kappa y+\lambda y^{3} \mathrm{e}^{-y^{2}}$

where the normalising coefficients are $\eta=0.9213$, $\kappa=-2.205$ and $\lambda=0.9780$ [4].

Most energy-based approaches suffer from scale problems for tuning the temporal width of the filters. Spatio-temporal filters used for computing optical flow require multiple banks at different scales [3]. However, we have found that using a temporal width of 10 frames for our sequences captured at $8 \mathrm{~Hz}$, provide acceptable, although not optimal, energy histories for a variety of events. Fig. 2 shows typical energy histories extracted from different pixels in another sequence containing 10 repetitions of a right-left-right movement by a person. Pixel-energy information can be seen to possess distinctive repeatable signatures caused by different patterns of change from the view-point of the pixels.

We have used the Gaussian mixture models of Section 2 to detect sections of slow change in the energy signal and segment the latter into discrete pixel-events of temporally contiguous fast-change with a common 1D connected component algorithm. The third column of Fig. 2 shows energy histories of fast-change segmented from the continuous energy signals. The discrete pixel-event energy signatures obtained for fixed pixels from a training sequence of 'normal' activities are used as models for classifying new activities as normal (known) or abnormal (unknown). Essentially, semantics are being tied to specific energy histories through supervised learning. Probabilistic trajectory matching provides the mechanism for recognising new observations from pre-learnt models [2,5,7]. The CONDENSATION framework provides the flexibility of recognising new signals with different temporal and amplitude scales. Multiple hypotheses are generated to match a backward window, from time $t$ to time $t-w$ for a window of size $w$, on the signal against template windows in the models. The propagation of random samples allows for concurrent hypotheses to be maintained while providing temporal and amplitude scaling for signalmatching cross-correlation flexibility.

More precisely, the matching hypothesis or state $s_{t}$ for a pixel at time $t$ is defined as $\left(\mu_{t}, \phi_{t}, \alpha_{t}, \rho_{t}\right)$ where $\mu$ is the model (pixel-event energy-history) being matched, $\phi$, the position of the correlation window in the model, $\alpha$ and $\rho$ are the amplitude and temporal scaling parameters, respectively, as shown in Fig. 3. A finite set of $k$ states are then propagated across time according to a cross-correlation observation probability as defined in Ref. [2]

$P\left(\mathbf{y}_{t} \mid \mathbf{s}_{t}\right)=\exp \left\{-\sum_{j=0}^{w-1} \frac{\left(\mathbf{y}_{t-j}-\alpha m_{(\phi-\rho j)}^{\mu}\right)^{2}}{2 \sigma_{\mu}(w-1)}\right\}$

States are randomly chosen from a cumulative probability distribution of the normalised observation probabilities of
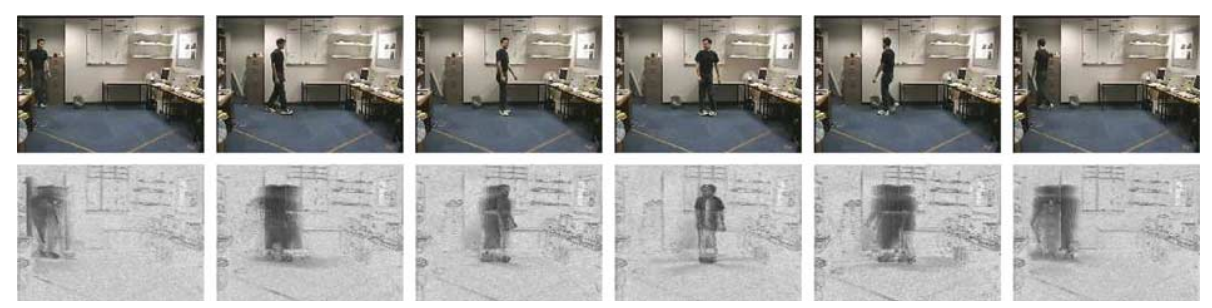

Fig. 1. Rows from top to bottom: (a) Selected frames from a right-left-right walking sequence. (b) Pixel-energy data is encoded in grey-level using a log-scale to show small scale structures. (Black indicates high response). Reflective edges can be seen with small responses. 

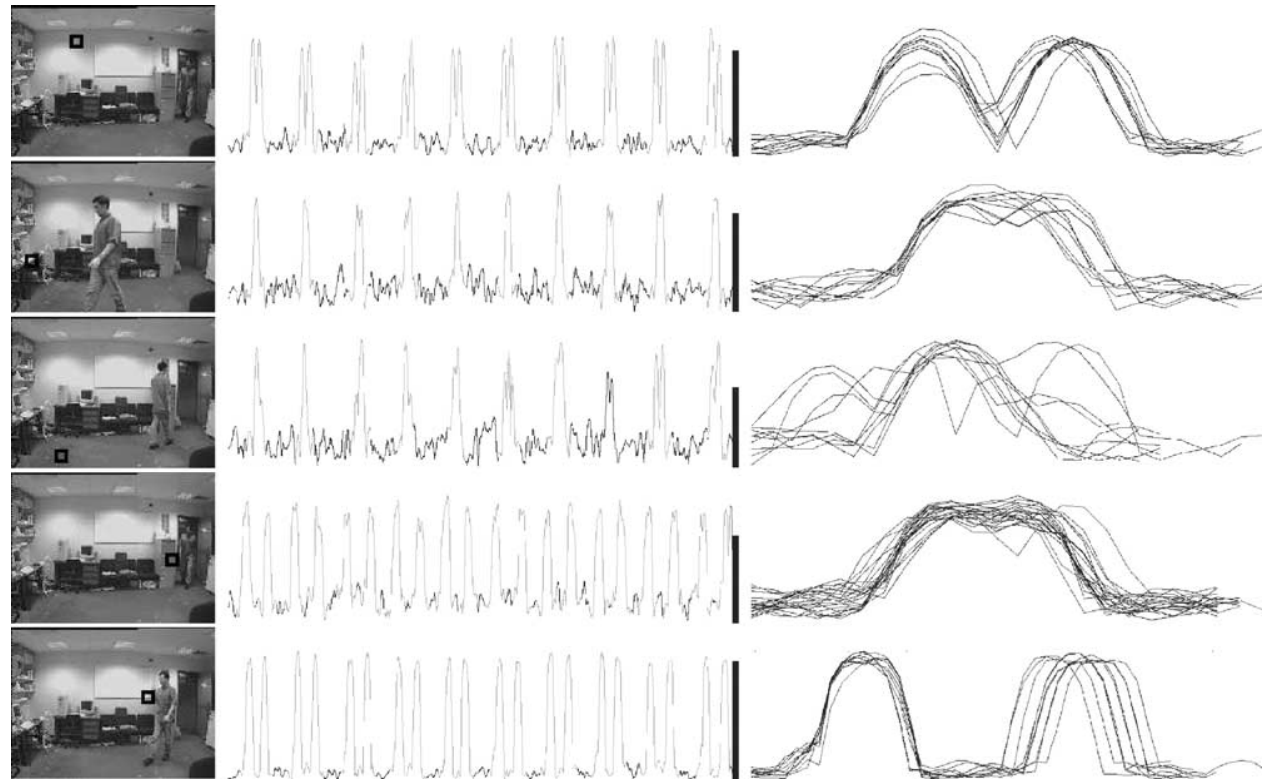

Fig. 2. From left-to-right. (a)Selected frames of a sequence containing 10 right-left-right movements are shown, with a black square for selected pixels. (b) Distinctive and repeatable structures from energy histories of the entire sequence for the pixel. Gray parts indicate fast-change while black parts indicate slow change, obtained form GMM (akin to background subtraction). (c) Normalised and super-imposed energy-histories of temporally contiguous fast-change which have been segmented from the previous column.

all the states in the set. Then, states with observation probability higher than a certain threshold of probable match (we use a threshold of $30 \%$ confidence) are propagated to the next time step according to

$$
\begin{aligned}
\mu_{t} & =\mu_{t-1} \\
\phi_{t} & =\phi_{t-1}+\rho_{t-1}+N \\
\alpha_{t} & =\alpha_{t-1}+N \\
\rho_{t} & =\rho_{t-1}+N
\end{aligned}
$$

where $N$ is added normal noise for performing local search in parameter space.

The propagative dynamics of the cross-correlation windows involve predicting pixel-energy values for the next time step from previously learnt energy models. Hypotheses therefore track a correlation feature space for single pixels, matching new signals against learnt models. However, recognising energy histories for single pixels can be prone to noise and can also suffer from ambiguities arising from spatio-temporal interference effects by textured surfaces. Without resorting to full spatio-temporal motion-energy filters, the effect of synchrony in visual information can be exploited. Global events affect multiple pixels simultaneously and irrespective of the type of textured change occurring at the object-level, the pixel-energy information of the involved stream of pixels should exhibit strongly correlated change. Preserving a common time reference for each learnt energy-history allows for synchronous cross-propagation of correlation matching hypotheses across pixels. A percentage of the states are reserved for random initialisation and cross-propagation. The propagative dynamics of the samples are upgraded as:

- Given a pixel $\mathbf{x}_{t}$ at time $t$, for all the samples $\left(\mu_{t}, \phi_{t}\right.$, $\alpha_{t}, \rho_{t}$ ) satisfying the matching confidence threshold, another pixel $\mathbf{y}_{t}$ with energy-history model $\mu_{t}^{\prime}$, which occurred at the same time as $\mu_{t}$ during training with corresponding model time index $\phi_{t}^{\prime}$, is selected. Assuming that the scaling required to match the learned models to the new observations applies across pixels, a new sample $\left(\mu_{t}^{\prime}, \phi_{t}^{\prime}+\rho_{t}, \alpha_{t}, \rho_{t}\right)$ is cross-propagated into pixel $\mathbf{y}_{t}$ at the next time step with similar amplitude and temporal scaling factors as the original sample.

The probability of the energy in a given pixel at time $t$ matching the pre-learnt normal models is given as the best

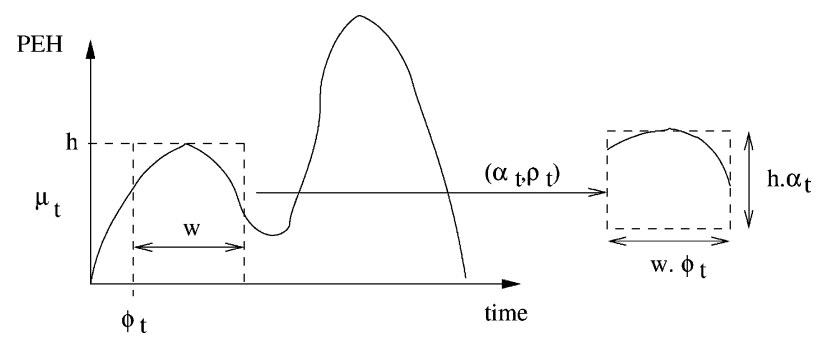

Fig. 3. A matching hypothesis or state $s_{t}$ consisting of parameters: model ( $\mu_{t}$, position $\phi_{t}$ of the matching window in the model, amplitude scaling $\alpha_{t}$, and temporal scaling $\rho_{t}$ ). 

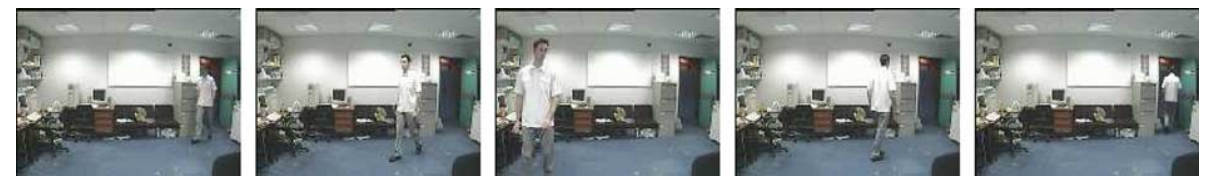

Fig. 4. Selected frames from the training sequence.

observation probability over a set of $k$ states

$P\left(\mathbf{y}_{t}\right)=\max _{i=1}^{k}\left(P\left(\mathbf{y}_{t} \mid \mathbf{s}_{i, t}\right)\right)$

While recognising patterns of pixel-change in a new sequence, the technique generates hypotheses of normal (known) energy histories matching energy data from the training sequence. Good hypotheses generate cross-hypotheses in other pixels, which are synchronously involved in similar fast-change during the learning stage. Events can therefore sustain adequate recognition by pixels crosspropagating hypotheses to each other and back. The technique provides a good alternative for learning the binding process of pixel-events into higher-level spatiotemporal events without object representations and the use of spatial proximity as a correlation criteria.

Table 1

Abnormal event detection results for the test sequences totalling over 1700 frames

\begin{tabular}{|c|c|c|c|c|c|c|}
\hline \multirow{2}{*}{$\begin{array}{l}\text { Test } \\
\text { events }\end{array}$} & \multirow{2}{*}{$\begin{array}{l}\text { No. of event } \\
\text { occurrences }\end{array}$} & \multirow{2}{*}{$\begin{array}{l}\text { No. of } \\
\text { frames }\end{array}$} & \multicolumn{2}{|l|}{ DEM } & \multicolumn{2}{|l|}{ GMM } \\
\hline & & & Detected & $\%$ & Detected & $\%$ \\
\hline $\begin{array}{l}\text { Slow } \\
\text { movement }\end{array}$ & 6 & 615 & 6 & 100 & 6 & 100 \\
\hline $\begin{array}{l}\text { Fast } \\
\text { movement }\end{array}$ & 6 & 255 & 6 & 100 & 6 & 100 \\
\hline $\begin{array}{l}\text { Stationary } \\
\text { pause }\end{array}$ & 6 & 362 & 5 & 83.3 & 0 & 0 \\
\hline Jump & 6 & 356 & 4 & 66.7 & 0 & 0 \\
\hline $\begin{array}{l}\text { Falling } \\
\text { box }\end{array}$ & 1 & 108 & 1 & 100 & 0 & 0 \\
\hline
\end{tabular}

This is based on the deviant-event detector's (DED) and GMM model's ability to correctly classify events containing normal and abnormal motion.
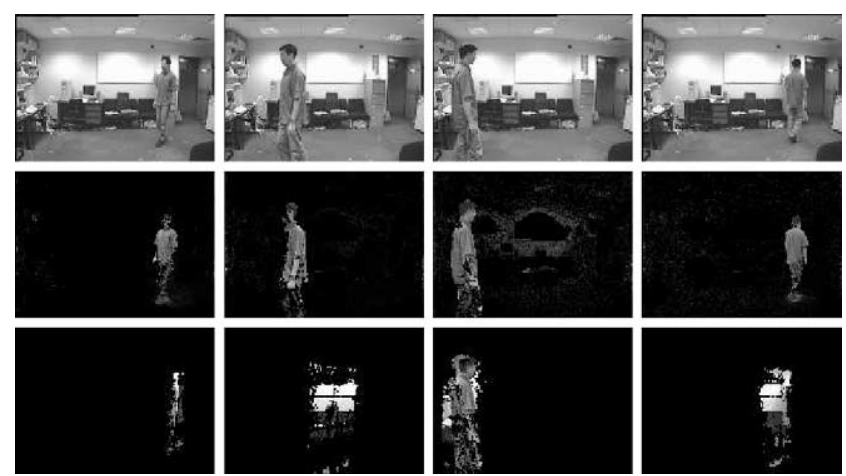

Fig. 5. Detection results for the 'Slow Movement' event. From top row to bottom: original images, GMM model detection and deviant-event detection.

\section{Experiments}

To illustrate how semantics can be incorporated into temporal models based on energy histories and how the learnt models can be used to detect unknown deviant events, we give some preliminary results. The system is trained on a sequence of approximately 1700 frames (from 20 repeated events) containing two people carrying out their normal routine of entering the office from the door on the right, moving to the left for an inspection and leaving by the same door, as in Fig. 4.

After training, the system was tested on five sequences of activities performed by three persons, one of whom was not present during training. The testing sequences contain similar events to the training sequences but with differences in the characteristics of performed movement so as to render either part of or the whole activity 'abnormal'. First, the test subject repeated the movement at (a) slower and (b) faster speeds. A stationary pause (c) and a quick jump (d) were introduced in the middle of the right-left movement. Finally, the system was retrained to include a static object (a box) in the lower right corner of the room. The context of the environment allows for movement by the person in the scene. However, the event of the box falling over (e) is not considered as normal.

Table 1 shows the results of deviant-event detection over the five test categories. For the 'Slow Movement' and 'Fast Movement' sequences, the deviant parts of the events have been successfully detected as shown in Fig. 5. The deviant-event detector (DED) perform better in sequences which involve semantically meaningful deviations from pre-learnt patterns of change, such as 'Stationary pause' and 'Jump' where only the deviant parts of the events are detected as shown in Figs. 6 and 7. As the Gaussian mixture models do not possess any
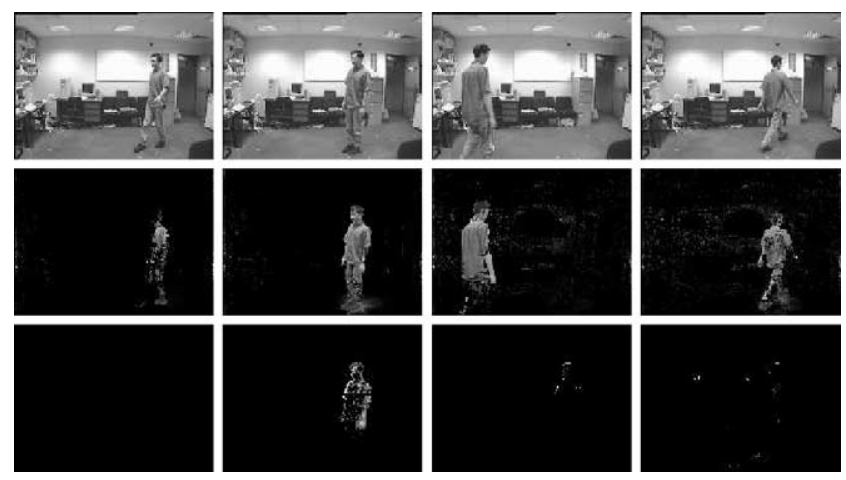

Fig. 6. Results from the 'Stationary Pause' event. 

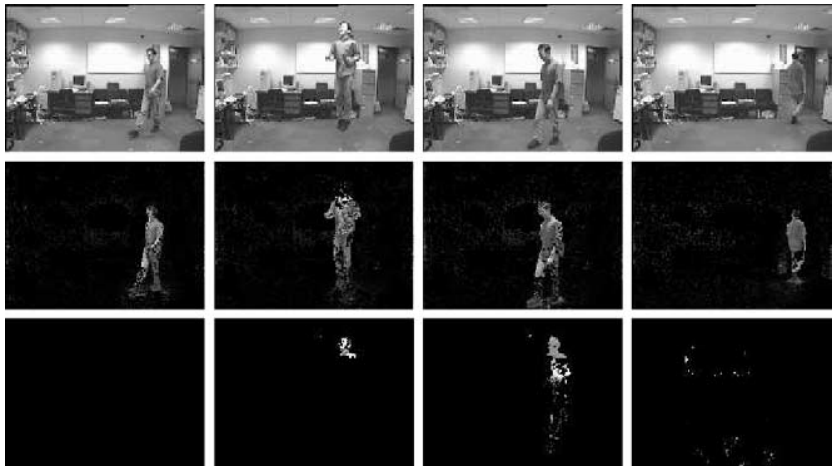

Fig. 7. Detection results from the 'Jump' event.

knowledge of context, they detect all movement as abnormal events. In the 'Falling box' sequence, the movement of the person is considered as normal in the particular context of that office environment as the DED correctly matches the per-pixel change occurring in the frames with its pre-learnt patterns. Both the DED and the Gaussian mixture model do detect the event when the box falls (Fig. 8).

The results show that general semantics concerning the type of change in individual pixels can be used to differentiate between different classes of events and indeed selectively identify locations and time in the scene where unknown deviant change occurred. Such ability provides

(a) Frames

(b) GMM Det.

(c) DEM Det.

t1

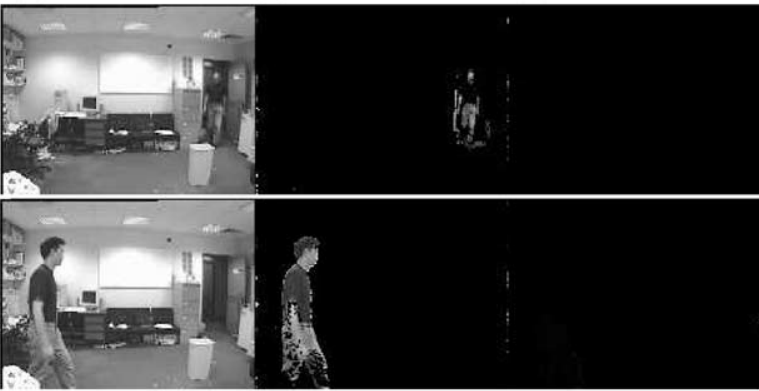

$\mathrm{t} 38$

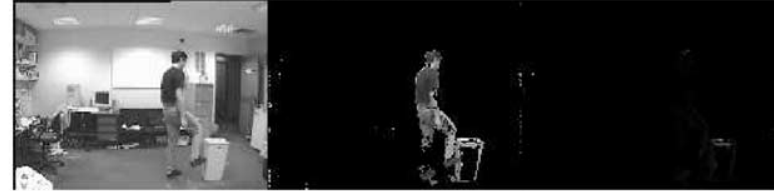

$\mathrm{t} 40$

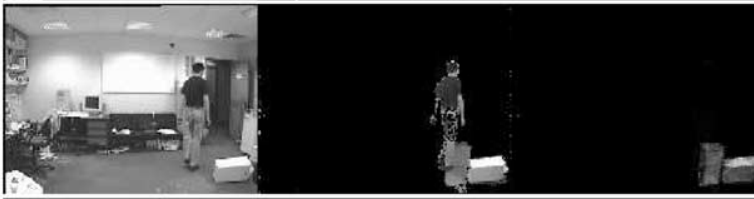

$\mathrm{t} 45$

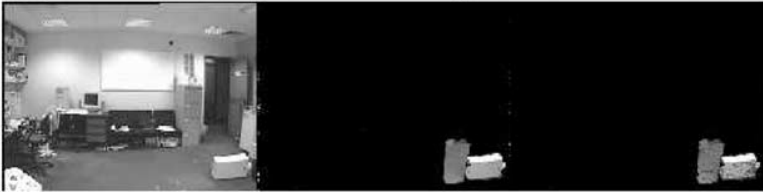

Fig. 8. Comparison of detections by a GM-based dynamic model (GMM) and our deviant-event detector (DED). additional flexibility over Gaussian mixture models for monitoring complex events in dynamic environments. Furthermore, the deviant-event system selectively detects only abnormal events and can therefore be used as a pre-attentive mechanism for initiating person or object tracking.

\section{Conclusion and future work}

Modelling behaviours and recognising events often require object-level representations to interpret visual data. However, object segmentation and trajectory extraction relies on spatial proximity (region-growing) and temporally constrained correlations. However, using such assumptions in busy scenes might not be sufficient. We propose a new low-level representation, which can be linked to semantics to understand events and perform abnormal event detection without making any assumptions about objects. Energy-histories provide a condensed variable-length representation of fast temporal change in single pixels. Preliminary results show that they can be used to semantically discriminate between events involving different pace as well as patterns of change. We have also used Gaussian mixture models to separately model and recognise slow change such as illumination cycles under a less computationally taxing framework. The ambiguity inherent in viewing a complex world through a single pixel has been addressed by using synchronous change in multiple pixels during events to perform pixel-stream hypotheses.

Although we have used supervised learning to introduce semantics in a low-level framework, unsupervised learning can be used to extract common patterns of change over long periods of time. Currently, the synchronous prediction and recognition of activity in streams of pixels have not been fully investigated. Cross-propagation of pixel-matching hypotheses attempt to recognise familiar patterns of synchronous change spatially across the image and over the short period of time that cross-propagation can be sustained. The higher-order correlation between synchronous pixel-change such as relative onset of the pixel-changes and relative phase of pixel-changes can offer better cues for inferring more complex semantics of the changes in the scene. Future work will concentrate on the propagative dynamics for generating hypotheses and augmenting the technique to discriminate more subtle deviant-event recognition.

\section{References}

[1] E.H. Adelson, J.R. Bergen, The plenoptic function and the elements of early vision, In Computational Models of Visual Processing, MIT Press, Cambridge, MA, 1991. 
[2] M.J. Black, A.D. Jepson, A probabilistic framework for matching temporal trajectories: condensation-based recognition of gestures and expressions, In European Conference on Computer Vision, Freiburg, 1998, pp. 909-924.

[3] O. Chomat, J. Crowley, A probabilistic sensor for the perception and the recognition of activities, In European Conference on Computer Vision, Dublin, 2000, pp. 487-503.

[4] W.T. Freeman, E.H. Adelson, The design and use of steerable filters, IEEE PAMI 13 (9) (1991) 891-906.

[5] S. Gong, M. Walter, A. Psarrou, Recognition of temporal structures: learning prior and propagating observation augmented densities via hidden Markov states, In IEEE International Conference on Computer Vision, Confu, 1999, pp. 157-162.

[6] D.J. Heeger, Optical flow using spatiotemporal filters, International Journal of Computer Vision 1 (1987) 279-302.

[7] M. Isard, A. Blake, Contour tracking by stochastic propagation of conditional density, In European Conference on Computer Vision, Cambridge, 1996, pp. 343-356.
[8] N. Johnson, D.C. Hogg, Learning the distribution of object trajectories for event recognition, Image and Vision Computing 14 (8) (1996) $609-615$.

[9] S.J. McKenna, S. Jabri, Z. Duric, H. Wechsler, A. Rosenfeld, Tracking groups of people, Computer Vision and Image Understanding 80 (1) (2000) 42-56.

[10] A. Oppenheim, R. Schafer, Digital Signal Processing, Prentice-Hall, Englewood Cliffs, NJ, 1975.

[11] Y. Raja, S.J. McKenna, S. Gong, Tracking and segmenting people in varying lighting conditions using colour, In IEEE International Conference on Automatic Face and Gesture Recognition, Nara, 1998, pp. $228-233$.

[12] C. Stauffer, W.E.L. Grimson, Using adaptive tracking to classify and monitor activities in a site, In IEEE Conference on Computer Vision and Pattern Recognition, Los Alamitos, USA, 1998, pp. 22-29.

[14] M. Usher, N. Donnelly, Visual synchrony affects binding and segmentation in perception, Letter to Nature 394 (1998) 179-182. 